Review

\title{
Advances in the in Vivo Raman Spectroscopy of Malignant Skin Tumors Using Portable Instrumentation
}

\author{
Nikolaos Kourkoumelis ${ }^{1, *}$, Ioannis Balatsoukas ${ }^{1}$, Violetta Moulia ${ }^{1}$, Aspasia Elka ${ }^{1}$, \\ Georgios Gaitanis ${ }^{2}$ and Ioannis D. Bassukas ${ }^{2}$
}

1 Department of Medical Physics, School of Health Sciences, University of Ioannina, 45110 Ioannina, Greece; E-Mails: ibalats@cc.uoi.gr (I.B.); biomoulia@yahoo.gr (V.M.); e.aspa@yahoo.com (A.E.)

2 Department of Skin and Venereal Diseases, School of Health Sciences, University of Ioannina, 45110 Ioannina, Greece; E-Mails: ggaitan@uoi.gr (G.G.); ibassuka@cc.uoi.gr (I.D.B.)

* Author to whom correspondence should be addressed; E-Mail: nkourkou@cc.uoi.gr; Tel.: +30-2651-00-7594; Fax: +30-2651-00-7854.

Academic Editor: William Chi-shing Cho

Received: 11 June 2015 / Accepted: 24 June 2015 / Published: 26 June 2015

\begin{abstract}
Raman spectroscopy has emerged as a promising tool for real-time clinical diagnosis of malignant skin tumors offering a number of potential advantages: it is non-intrusive, it requires no sample preparation, and it features high chemical specificity with minimal water interference. However, in vivo tissue evaluation and accurate histopathological classification remain a challenging task for the successful transition from laboratory prototypes to clinical devices. In the literature, there are numerous reports on the applications of Raman spectroscopy to biomedical research and cancer diagnostics. Nevertheless, cases where real-time, portable instrumentations have been employed for the in vivo evaluation of skin lesions are scarce, despite their advantages in use as medical devices in the clinical setting. This paper reviews the advances in real-time Raman spectroscopy for the in vivo characterization of common skin lesions. The translational momentum of Raman spectroscopy towards the clinical practice is revealed by (i) assembling the technical specifications of portable systems and (ii) analyzing the spectral characteristics of in vivo measurements.
\end{abstract}

Keywords: Raman spectroscopy; in vivo spectroscopy; skin cancer 


\section{Introduction}

The spectrum of skin cancers in humans currently encompasses the most frequent neoplasm types by tissue of origin and the most costly cancer categories to treat [1]. From the different types of skin neoplasms, cancers of keratinocytic origin (epithelial skin cancer, formerly designated preferentially as non-melanoma skin cancer (NMSC)) form the most frequent category. The two most common skin cancer types of this group, basal cell carcinoma (BCC) and squamous cell carcinoma (SCC) comprise $\sim 95 \%$ of all skin cancers and together with the third most frequent, malignant melanoma (MM), constitute $99 \%$ of the incidence of all skin neoplasms. Several studies have documented the increasing trends in skin cancer occurrence all over the world [2-7]. Furthermore, distinct subpopulations exist with significantly increased risk for developing tumors in this organ like patients with multiple nevi [8] and those that are on long-term iatrogenic immunosuppresion [9]. However, the aforementioned three most frequent skin cancer types, i.e., BCC, SCC and MM, differ substantially in their biological aggressiveness and relevant prognosis. Keratinocytic skin cancers are typically curable, especially if diagnosed early enough [10] and despite the increasing incidence, their mortality rates remain low $[11,12]$. Notably for BCC, there are reports of rapidly declining mortality rates despite the marked incidence increase during the same period [13]. On the other hand, MM is a biologically aggressive neoplasm. The diagnosis is often ambiguous and the course of the disease strongly depends on the tumor stage [14]. In general, it is highly unpredictable at the individual patient's level; the prognosis is markedly worse in advanced stages with the five-year survival rate as low as $16 \%$. However, when melanoma is detected and excised in the initial in situ stage, survival reaches $99 \%$ and the disease can be considered practically curable [15].

Early diagnosis is critical for the successful treatment of skin neoplasms. The initial step of clinical evaluation in which suspicious lesions are selected for targeted invasive assessment with biopsies is crucial because the selection process is challenging even for the experienced clinician. Few lesions have to be picked up for biopsy between a multitude of benign and malignant and look-alike skin alterations coexist side by side in skin areas severely damaged by excessive life-long sunlight exposure. The specificity and the sensitivity of the above clinical procedure vary from $40 \%$ to $80 \%$, imposing substantial uncertainty on this diagnostic process [16]. Moreover, it is very important to limit the use of skin biopsies since this invasive and time-consuming process is often associated with substantial patient discomfort. At this point, unbiased clinical decisions based on non-invasive, real-time and time-saving diagnostic techniques are preferable to facilitate the management of the increasing number of patients with skin cancer [17,18]. From the many optical spectroscopy techniques under evaluation for the early, non-invasive detection of skin lesions, two vibrational spectroscopy modalities seem to be the most promising: Fourier Transform Infrared (FTIR) and Raman Spectroscopy [16,19,20]. The strong absorption of mid-infrared radiation by water molecules limits the clinical in vivo applicability of the FTIR technique [21], although a lot of progress has been achieved towards this issue [22]. On the other hand, Raman spectroscopy is able to detect spectroscopic fingerprints of tissues in their native state within clinically acceptable measurement time (seconds) without the need for tissue pre-processing, like staining or fixation.

Raman spectroscopy is based on the inelastic scattering of photons exchanging energy via molecular vibrational modes. Due to the fact that molecular energy levels are quantized and unique for 
each molecule, Raman spectra feature discrete and chemical bonds-specific bands, providing a "molecular fingerprint" of the samples under study [23]. Thus, Raman active molecules (i.e., with anisotropic polarizability) can give spectroscopic signals with significant information about the chemical composition of the sample. The technique has been applied in a variety of biological and medical research branches as a reliable modality for the in situ diagnosis of malignancy at tissue level or for the analysis of subcellular molecular composition of tissues [24]. Moreover, it can be employed to determine steps in tumor progression or to evaluate response to radiation therapy [25].

This paper reviews the advances in the use of portable Raman systems for the clinical, in vivo spectroscopic characterization of skin lesions in real-time with emphasis in the diagnosis and management of the three most common skin cancers (BCC, SCC and MM). Where possible, we compare the experimental outcomes in an effort to identify the best practices for the clinical environment.

\section{Instrumentation and Experimental Considerations}

\subsection{Portable Raman Acquisition Systems}

Raman spectroscopy is an optical spectroscopic technique based on the inelastic scattering of monochromatic light. Raman scattering involves inelastic collisions between the photons of an irradiating laser beam and the sample (or tissue) molecules. The absorption of photons, results in energy exchange between photons and tissue molecules generating vibrations within the material that are molecule specific. A nonlinear polyatomic molecule with $\mathrm{N}$ atoms has $3 \mathrm{~N}-6$ modes of vibration, known as "normal modes" which are related to a fundamental frequency of vibration and symmetry. When photons are reemitted, their energy, and hence frequency, is shifted in comparison to the excitation frequency, yielding the so-called "Raman shift". The Raman shift is independent of the excitation (incident) wavelength, which means that it is constant and unique for the different molecules. Thus, the evaluation of the scattered light is transformed to the vibrational Raman spectrum of the sample, which contains substantial qualitative and quantitative information about the chemical composition of the examined probes. Since the detection of certain Raman bands correlates with the presence of specific molecules in a probe, Raman spectra can be used to differentiate between tissues of different pathological conditions. Raman cross section is wavelength dependent and varies according to $1 / \lambda^{4}$, where $\lambda$ is the excitation wavelength [26]. Thus, the intensity of the Raman peaks is proportional to $\mathrm{f}^{4}$ (where $\mathrm{f}$ is the laser frequency) and, for example, a $488 \mathrm{~nm}$ laser gives an almost seven times more intense Raman signal than a $785 \mathrm{~nm}$ laser. Moreover, the transmission efficiency of the optics and detector sensitivity is also wavelength dependent [27]. The excitation wavelength is a significant parameter for the analysis of skin lesions because it has a major impact on the scattered intensity, the auto-fluorescence and the signal attenuation. Skin research can be conducted in a wide range of excitation frequencies from visible to near infrared (NIR) wavelengths and this has been an obstacle to compare methodologies and results. NIR generate lower auto-fluorescence intensities compared to the excitation with visible light but at the same time poorer scattering intensity too, resulting into lower sensitivity and thus to a lower signal to noise ratio. Laser sources at 785 or $830 \mathrm{~nm}$ are preferred for clinical Raman applications because the operation at these wavelengths combines reduced auto-fluorescence and adequate tissue penetration depth [28]. Sources at $1064 \mathrm{~nm}$ also have 
a favorable profile of signal to noise ratio for skin applications but they usually require increased irradiation doses and annoying prolonged spectra acquisition times [29,30]. Nevertheless, optimally employed FT-Raman spectrometers at this excitation wavelength demonstrate relatively high signal to noise ratio [31]. The light attenuation in the skin tissue was evaluated on excised human skin by confocal Raman microspectroscopy [32-35] and confirmed that Raman signals are suitable for skin measurements at various depths (up to a few hundred micrometers) depending on the spectrometer setup. The safety issues of tissue irradiation and exposure times are regulated by consensus directives released by international organizations (ANSI Z136, ICNIRP, and IEC 60825-1).

Table 1 summarizes the major operation parameters (wavelength, power, focal spot size, and signal integration time) employed in in vivo studies of a variety of skin lesions with the use of portable Raman spectroscopy devices for the acquisition of spectra in real-time (the blue line separates in vivo studies which did not aim to the classification of common skin lesions but solely to biochemical characterization). In the clinical setting with the appropriate equipment, Raman spectroscopy does not require any specific tissue pre-treatment except from possible superficial cleaning of excess sebum with ethanol [16]. Ex vivo studies of skin tissue material samples and biopsy probes are not included in Table 1, even if they have been carried out with portable setups.

The diverse Raman implementations presented in Table 1 imply that there is neither any optimal experimental design nor any standardization among setups. The data clearly indicate that it is difficult to quantify the tradeoff between excitation wavelength and lower scattering cross section with auto-fluorescence intensity since the detection of a specific Raman band depends on both the experimental parameters and the (heterogeneous) composition of skin tissue. Tfayli et al. [28] have studied the wavelength effect on pig skin epidermis by recording spectra at 532,633, and $785 \mathrm{~nm}$ and analyzing the variability and the repeatability of the experiments along with the effect of exposure time and in-depth signal attenuation. They suggested that the excitation wavelength at $785 \mathrm{~nm}$ is advantageous (compared to 532 and $633 \mathrm{~nm}$ ) for the fingerprint skin region, having lower signal attenuation. Yet, in principle, it is rather difficult to extrapolate outcomes and directly compare results by different research groups using different wavelengths and instrumentation. Varying sampling rates and different statistical classification schemes add another level of complexity for the preparation of an accepted clinical protocol. Calibration of portable Raman systems with standard samples of exact composition, resembling human skin, is possibly a requirement for the future. 
Table 1. Technical details of clinical applications of portable Raman spectroscopy in skin cancer diagnosis.

\begin{tabular}{|c|c|c|c|c|c|c|c|}
\hline Cancer Type & Technique & $\begin{array}{l}\text { Raman Excitation } \\
\text { Wavelength (nm) }\end{array}$ & $\begin{array}{l}\text { Spot Size } \\
(\mathbf{m m})\end{array}$ & $\begin{array}{l}\text { Power } \\
(\mathrm{mW})\end{array}$ & $\begin{array}{c}\text { Signal Integration } \\
\text { Time (s) }\end{array}$ & $\begin{array}{l}\text { Number of Skin Lesions } \\
\text { Studied and/or Patients }\end{array}$ & Reference \\
\hline $\begin{array}{l}\text { MM, BCC, SCC, actinic keratosis (AK), atypical nevi, } \\
\text { melanocytic nevi, blue nevi, and seborrheic keratoses }\end{array}$ & Raman & 785 & 3.5 & 300 & 1 & 518 (453 patients) & {$[36]$} \\
\hline $\mathrm{BCC}$, inflammatory scar tissues & Raman + OCT $^{\text {a }}$ & 785 & 0.044 & 40 & 30 & 1 patient & {$[15]$} \\
\hline MM, BCC, SCC, pigmented nevi & Raman & 785 & 1 & 150 & 30 & 50 & [37] \\
\hline MM, BCC, SCC, pigmented nevi & Raman + OCT & 785 & 1 & 150 & 30 & 23,50 & {$[38,39]$} \\
\hline MM, BCC, SCC, pigmented nevi & Raman & 785 & 0.1 & 17 & 10 & 137 & {$[40,41]$} \\
\hline BCC, SCC, inflammatory scar tissues & Raman & 825 & $0.005^{b}$ & 40 & 30 & 21 (19 patients) & {$[42]$} \\
\hline $\mathrm{BCC}$ & Raman & 830 & 1.6 & 110 & 30 & 10 patients & {$[43]$} \\
\hline $\mathrm{BCC}, \mathrm{SCC}$ & Raman & 830 & - & 200 & $20(2 \mathrm{~s} \times 10$ spectra $)$ & 31 (17 patients) & {$[44]$} \\
\hline $\mathrm{BCC}, \mathrm{SCC}, \mathrm{AK}$ & Raman & 830 & 0.17 & 200 & $20(2 \mathrm{~s} \times 10$ spectra $)$ & 49 (25 patients) & {$[45]$} \\
\hline MM, BCC, SCC, AK, and non-melanoma pigmented lesions & Raman & 830 & 0.2 & 100 & 1 & 137 (76 patients) & {$[46,47]$} \\
\hline $\mathrm{BCC}$ & Multi modal ${ }^{\mathrm{c}}$ & 830 & 0.2 & 56 & 4 & 1 (healthy) ${ }^{d}$ & [48] \\
\hline MM, eczema, psoriatic skin, malignant Kaposi sarcomas & Raman & 1064 & 10 & - & - & 1 (healthy) ${ }^{d}$ & [31] \\
\hline MM, BCC, pigmented nevi & Raman & 1064 & 0.1 & 120 & 480 & 81 (72 patients) & [49] \\
\hline Carotenoid concentration in $\mathrm{BCC}$ and actinic keratoses & Raman & 488 & 2 & 10 & 20 & 14 patients & {$[50]$} \\
\hline MM & Multi modal ${ }^{\mathrm{e}}$ & 1064 & 0.08 & - & 35 & Mice injected with human MM cells & {$[51]$} \\
\hline
\end{tabular}

${ }^{\mathrm{a}}$ OCT = Optical Coherence Tomography; ${ }^{\mathrm{b}}$ Value from identical instrumentation in [52]; ${ }^{\mathrm{c}}$ Raman, fluorescence, and reflectance spectroscopy; ${ }^{\mathrm{d}}$ compared with skin lesions from in vitro studies; ${ }^{\text {e }}$ acoustic microscopy, infrared reflectance and Raman spectroscopy (proof-of-concept study); BCC: basal cell carcinoma; SCC: squamous cell carcinoma; MM: malignant melanoma. 


\subsection{Comparison between Portable and Benchtop Systems}

As a rule of the thumb, the spectral quality of portable spectrometers is lower than benchtop ones due to the poorer sensitivity and scanning range [53]. However, the superior characteristics of the benchtop spectrometers (numerical aperture, lens, and focal length) and of the laser beam (intensity profile) are compensated by mass and size restrictions in the clinical environment along with the convenient accessibility of the skin lesions on the patients' bodies via optical fibers. In most cases, bands assignment and qualitative characterization was aided by measurements performed in biopsies and no significant alterations in bands shape and positions has been detected between in vivo and in vitro experiments. Even so, the overall spectra precision was characterized as low [54] due to the low signal to noise ratio. Thus, portable Raman setups should be able to address the question whether or not a band having 2-3 times greater intensity than the noise (which is typically defined as the square root of the raw intensity of measurement) can be detected at a certain position [27]. Raman signal is effectively the sum of two parts: the raw signal attributed to the sample's concentration of Raman active compounds and the overall noise due to (i) fluorescence (background) and (ii) the contribution from the optical components. In addition, the resulted spectra are affected by ambient light, beam powder density, measurement time and matrix effects. Biomolecules in skin tissues are by default weak Raman scatterers imposing an additional difficulty to the challenging task of skin tumors classification. In the majority of the relevant literature, the application of chemometrics was necessary to detect the subtle changes attributed to the pathological skin lesions.

\section{Results and Discussion}

\subsection{Bands Assignment}

The positions (typically reported in wavenumbers) and relative intensities of Raman bands are the major spectral characteristics for delineating the molecular background of skin diseases. Nonetheless, for optimizing the extraction of diagnostic information, three interwoven but distinct levels of spectral analysis must be combined [55]: (i) chemical analysis which pertains to the qualitative and quantitative biochemical composition of the tissue; (ii) statistical processing of the spectral variations; and (iii) integration of core spectroscopic findings with relevant clinical information. The most common Raman bands associated with pathological skin lesions together with their chemical assignment are shown in Table 2. 
Table 2. Tentative assignment of the most prominent Raman bands showing differentiation in spectra between normal and skin cancer tissues (MM, BCC, and SCC specific).

\begin{tabular}{|c|c|c|c|}
\hline Cancer Type & Peak Position $\left(\mathrm{cm}^{-1}\right)^{a}$ & Assignment & Reference \\
\hline $\mathrm{BCC}$ & $500-600$ & S-S disulfide stretching & [56] \\
\hline $\mathrm{BCC}$ & 727 & $\mathrm{v}(\mathrm{CN})$ Adenine, Lipids & [56] \\
\hline $\mathrm{BCC}$ & 746 & Thymine & [56] \\
\hline $\mathrm{BCC}$ & $786-788$ & Nucleic acid backbone $\left(\mathrm{PO}_{2}\right.$ symmetric stretching) & {$[56,57]$} \\
\hline $\mathrm{BCC}, \mathrm{SCC}, \mathrm{MM}$ & 832 & $\begin{array}{l}\text { Proline, hydroxyproline, tyrosine, stretch of nucleic acids, } \\
\text { DNA }\left(\mathrm{PO}_{2} \text { symmetric stretching }\right)\end{array}$ & {$[44,46,56]$} \\
\hline $\mathrm{BCC}, \mathrm{SCC}$ & $920-943$ & $\mathrm{v}(\mathrm{CC})$ skeletal of collagen backbone Proline, hydroxyproline & {$[31,42,44,58]$} \\
\hline $\mathrm{BCC}, \mathrm{SCC}$ & $1000-1010$ & Phenylalanine (ring breathing); keratin & {$[42,44,56]$} \\
\hline $\mathrm{BCC}, \mathrm{SCC}$ & $1085-1098$ & $\mathrm{v}(\mathrm{CC})$ lipids Nucleic acid backbone $\mathrm{v}\left(\mathrm{PO}_{2}\right)$ symmetric stretching & {$[15,31,56,57,59]$} \\
\hline $\mathrm{BCC}, \mathrm{SCC}$ & $1127-1130$ & Lipids $\mathrm{v}(\mathrm{CC})$ symmetric stretching of acyl-backbone, trans conformation & {$[44,56]$} \\
\hline $\mathrm{BCC}, \mathrm{SCC}$ & $1207-1209$ & Tyrosine, phenylalanine & {$[44,56]$} \\
\hline $\mathrm{MM}, \mathrm{BCC}$ & $1220-1280$ & $\begin{array}{l}\text { Amide III ( } \delta(\mathrm{NH}) \text { bending and } v(\mathrm{CN}) \text { stretching vibrations) (protein band), } \\
\text { tropocollagen (proline-rich), } \mathrm{v}(\mathrm{CH}) \text { ethylene (triolein and phospholipids) }\end{array}$ & {$[15,31,42,43,45,46,49,56,58-61]$} \\
\hline $\mathrm{MM}, \mathrm{BCC}$ & 1300 & $\delta\left(\mathrm{CH}_{2}\right)$ twist, lipids & {$[46,60,62]$} \\
\hline $\mathrm{BCC}$ & 1336 & $\mathrm{CH}$ deformations, adenine, phenylalanine & {$[37,38,56]$} \\
\hline $\mathrm{MM}, \mathrm{BCC}$ & $1440-1460$ & $\delta\left(\mathrm{CH}_{2}\right)$ scissoring in lipids and $\delta\left(\mathrm{CH}_{2}\right)$ scissoring vibration in proteins & {$[15,42,46,56,59,62]$} \\
\hline $\mathrm{MM}, \mathrm{BCC}$ & $1520-1570$ & Nucleic acids & [37] \\
\hline $\mathrm{MM}, \mathrm{BCC}$ & $1640-1685$ & Amide $\mathrm{I}(\mathrm{C}=\mathrm{O}$ stretching), collagen, elastin & {$[37,38,42,43,46,59,60]$} \\
\hline $\mathrm{BCC}$ & 1651 & Lipids $(\mathrm{C}=\mathrm{C}$ stretching), phenylalanine & [56] \\
\hline $\mathrm{MM}, \mathrm{BCC}$ & 3250 & $\mathrm{H}_{2} \mathrm{O}$ & [49] \\
\hline
\end{tabular}

\footnotetext{
${ }^{\text {a }}$ Approximate values.
} 
Data in Table 2 indicate that quantitative modulations of amide moieties bands III and I are characteristic for tumor cell proliferation, probably as a result of Raman active modifications in the molecular composition of the proteins in malignant skin tissues. In particular, the majority of the studies report a significant decrease of the amide III band intensity, which is regarded to reflect important alterations in the secondary structure of the tissue proteins. It is important to note that this band gives quite strong signal with Raman but rather weak with IR spectroscopy [63]. Apart from reduced intensity, this band seems to also be shifted to higher frequencies in the case of BCC and MM [59]. Raman spectra from malignant skin lesions show lower amide I band intensities although the corresponding results are possibly tumor type specific. Significant changes are also observed in the lipids concentration. Moreover, it is expected that the concentration of nucleic acids increases in malignant tissues due to the unrestrained cell proliferation that leads to spatial overcrowding of nucleated cells $[31,64]$ and to intensity increase of the corresponding Raman bands. Water is another major tissue constituent. Highly significant alterations in the respective water content were observed for MM and BCC [49]. Spectral fingerprints of stabilizing disulfide bridges between cysteine residues in macromolecules are localized in the lower spectra section; detection of decreased intensity of the respective bands in tissues has been attributed to protein oxidation [56]. Carotenoids play a significant role as major anti-oxidants in the skin and they possibly contribute to the mechanisms of skin cancer prevention. Hata et al. [50] studied the concentration of carotenoids in the skin by analyzing the intensity of the $1524 \mathrm{~cm}^{-1}$ peak $(\mathrm{v}(\mathrm{C}=\mathrm{C})$ stretch vibration of the backbone). They suggested that $\mathrm{BCC}$ can be differentiated from healthy skin areas as a result of their significantly lower carotenoid concentration but at the same time they concluded that further analysis on the possible correlation of the dermal carotenoids with cutaneous pathology is needed. Indeed, recent studies using resonance Raman spectroscopy [65] and reflection spectroscopy [66] showed that carotenoids concentration mostly reflects nutritional habits and stress conditions. Poor nutrition, illness, and smoking are generally related to low carotenoid concentration while stress factors (solar irradiation, environmental hazards, fatigue, illness, etc.) contribute to the fast degradation of dermal carotenoids. Therefore, the low carotenoids concentration is undoubtedly a significant finding but probably not a biomarker.

It is evident that certain biochemical changes associated with skin malignancies can be successfully identified by Raman spectroscopy. To reduce the high number of parameters needed to characterize the variance in the acquired spectral datasets, researchers typically utilize multivariate statistical methods to generate linear discriminant models of classification [67]. In the subsequent section we also refer to the sensitivity and specificity of these models for BCC, SCC and MM.

\subsection{Studies of NMSC and MM with Portable Raman Instrumentation}

Liu et al. [36] performed the most comprehensive in vivo study to date presenting data from Raman spectroscopic analysis of 453 patients with benign and malignant skin lesions (MM, BCC, SCC, and actinic keratoses). They concluded that distinctive Raman spectral peaks or bands cannot be uniquely assigned to any of the different skin cancer types. Moreover, they showed that the chemical fingerprints of different skin lesions are not site-dependent unlike previous findings with healthy human skin [68]. Raman spectral bands with diagnostic impact were recognized at 855, 936, 1002, $1271,1302,1445,1655$, and $1745 \mathrm{~cm}^{-1}$. It was suggested that bands in the region of $1055-1800 \mathrm{~cm}^{-1}$ 
are critical for evaluating MM. Multivariate statistical methods were necessary to evaluate information from multiple band positions determinations in order to optimize the clinical classification of skin lesions. Although the sensitivity of the above approach for the discrimination between malignant and benign skin conditions is $99 \%$, the specificity is limited to only $15 \%$ for the differentiation between $\mathrm{MM}$ and benign lesions and to $17 \%$ for that of keratinocytic skin neoplasms (BCC, SCC and actinic keratoses) vs. benign lesions. Lim et al. [46] have noticed a noteworthy low intensity of the amide band I region coupled with an increase in the intensity of the lipid bands at $1310-1340 \mathrm{~cm}^{-1}$ in the case of MM. The decrease in the amide I band was linked to collagen degradation or to the increased melanin concentration and pigmentation of the neoplasms. Moreover, only the MM spectra exhibited peaks between 800 and $900 \mathrm{~cm}^{-1}$ while both BCC and MM showed peaks with lower intensity in the $1450 \mathrm{~cm}^{-1}$ region. The integration of spectral information in the fingerprint region $\left(900-1800 \mathrm{~cm}^{-1}\right)$ was suggested for the discrimination of skin cancers from benign lesions (Figure 1).
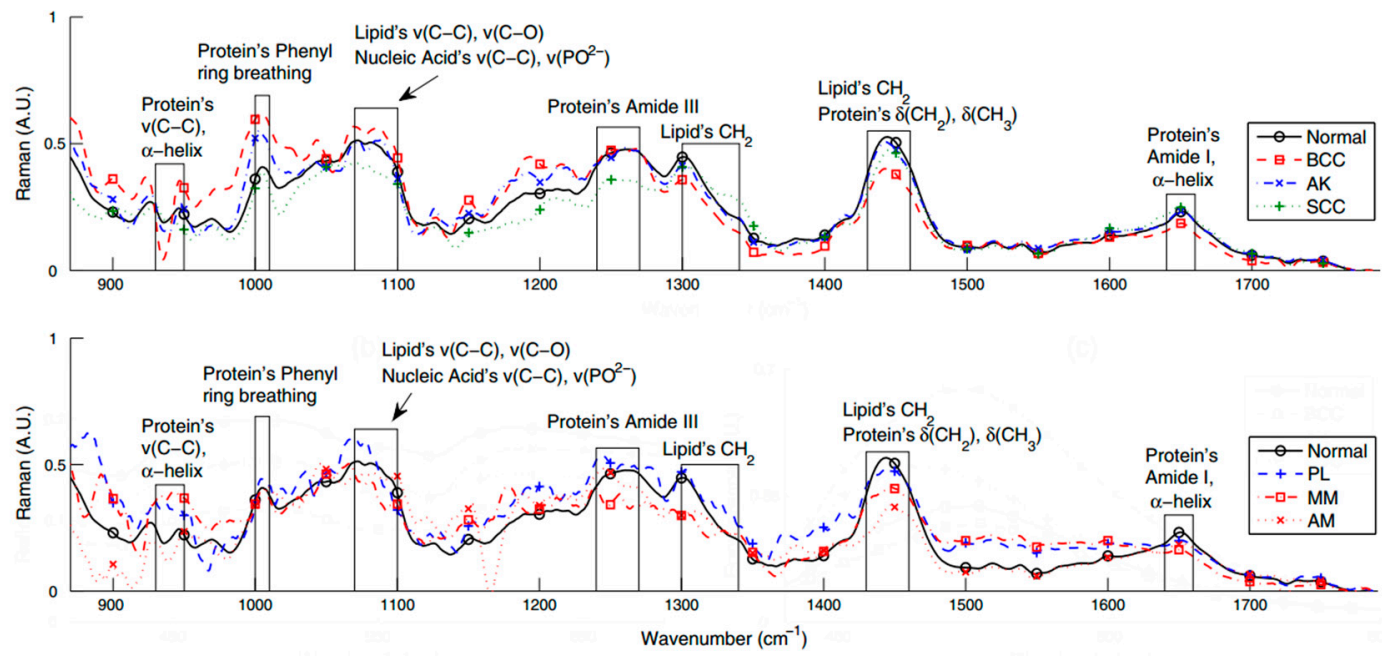

Figure 1. Mean Raman spectra acquired in vivo for basal cell carcinoma (BCC), squamous cell carcinoma (SCC), actinic keratosis (AK), nonmelanoma pigmented lesions (PL), amelanotic melanoma (AM) and normal skin. Adapted and reproduced with permission from [46].

The overall lower spectral intensity of malignant skin conditions was also confirmed by other studies [69], which consistently found that BCC and SCC exhibit weaker scattering than healthy skin, probably as a result of enhanced collagen breakdown. Principal component analysis (PCA) was employed for the classification of the different lesions. With this approach the authors demonstrated a $100 \%$ sensitivity and specificity in the discrimination of MM from non-melanoma pigmented skin lesions. With the same approach SCC, BCC and actinic keratoses could also be differentiated from normal skin with sensitivity and specificity of $90 \%$ and $85 \%$, respectively. Patil et al. [15] identified distinct differences between the healthy skin and BCC in the Raman bands at 1090, 1300, and $1440 \mathrm{~cm}^{-1}$ while Tfayli et al. [43] for the same kind of comparison (normal skin $v s$. BCC) performed hierarchical cluster analysis concentrating on the amide III and the $\mathrm{CH}$ deformation band $\left(1410 \mathrm{~cm}^{-1}\right)$ as the most promising bands; they noticed frequency shifting of the $1410 \mathrm{~cm}^{-1}$ peak, a weak shoulder at $1685 \mathrm{~cm}^{-1}$ in the BCC spectra (amide I region), differences in the amide III band and in the phospholipid content. Zakharov et al. [37] investigated major spectral differences between normal skin 
and malignant tumors ( $\mathrm{MM}$ and $\mathrm{BCC}$ ) using a two-step method of tumor diagnosis to optimize the sensitivity and specificity of the discrimination. The method utilized characteristic spectral intensities in the 1300-1340, 1440-1460, and 1640-1680 $\mathrm{cm}^{-1}$ ranges. Plots of intensities' relative difference were constructed for healthy and malignant tissues; discriminant analysis and support vector machines assigned tissue classes with $88.9 \%$ sensitivity and $87.8 \%$ specificity for the in vivo Raman measurements. The authors reported a significant improvement in MM identification using this two-step classification. The same research group enhanced their technique by combining Raman spectroscopy with OCT [38] and in a recent publication, combined OCT, backscattering (BS) and Raman spectroscopy [39]. OCT is a highly accurate method for BCC diagnosis while BS was utilized for the qualification of tumor boundaries. This combined device coupled with the two-step analysis showed an increase accuracy of diagnosis by $9 \%$ for sensitivity and $8 \%$ for specificity compared to the values obtained by each method separately. Comparative results for BCC vs. normal tissues are depicted in Figure 2. Common significant spectral features are concentrated in the amide III and lipids bands while the band at $1410 \mathrm{~cm}^{-1}$ was not reported in studies other than [43]. The weak shoulder at $1685 \mathrm{~cm}^{-1}$ is present in two of the studies [15,43] while it was not evident in the third one [37]; this possibly shows that amide I alterations are tumor specific and readily expressed in MM but not in BCC.

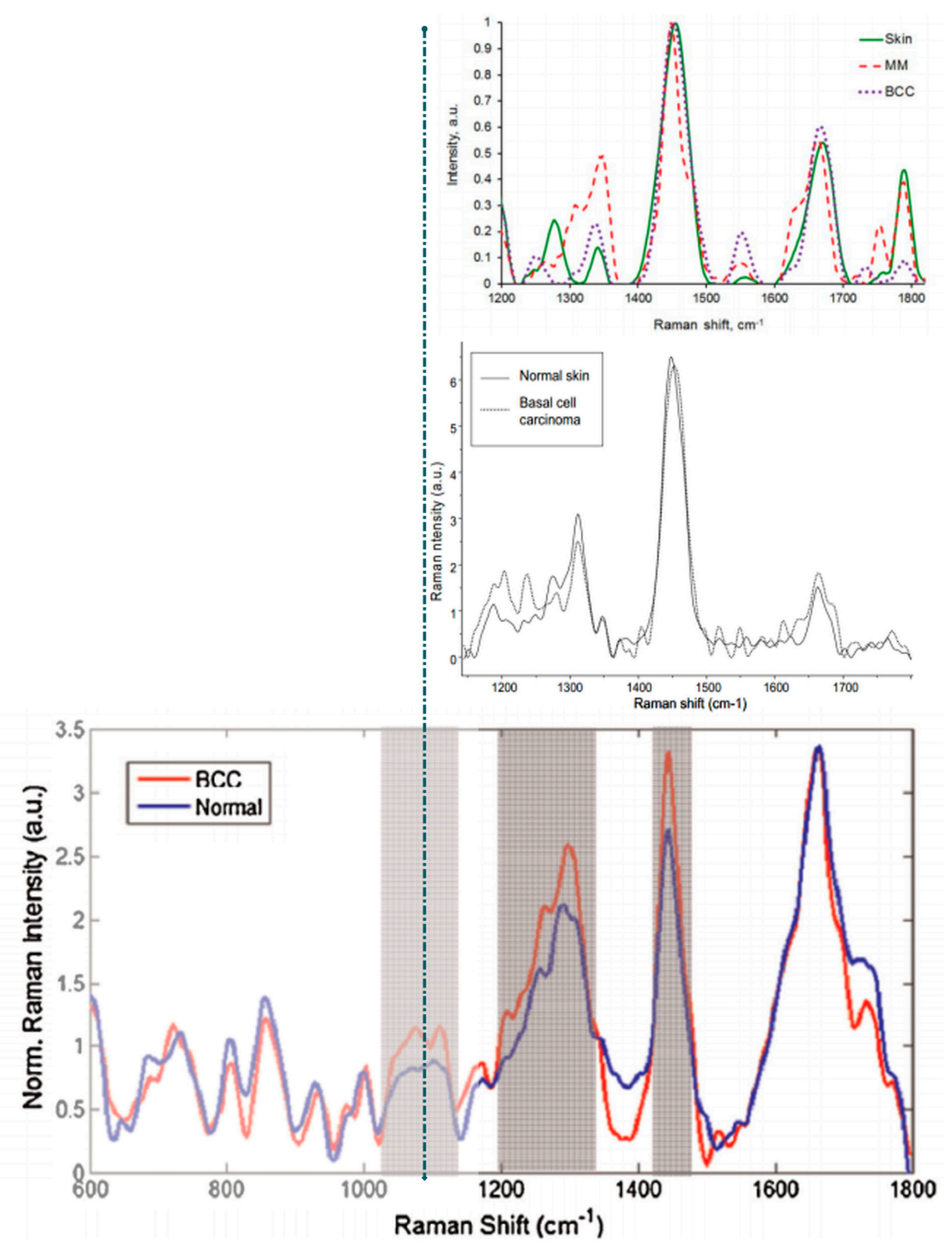

Figure 2. Comparative results (from three research groups) of in vivo Raman spectra for BCC and normal skin. Adapted and reproduced with permission from [15,37,43]. 
Amide III band also exhibited blue-shift and intensity decrease in in vitro studies [59], consistent with secondary protein structure changes in BCC. Moreover, it was shown that the $\mathrm{CH}_{2}$ bending mode (lipids and protein) was shifted to higher frequencies and the $\mathrm{v}\left(\mathrm{PO}_{2}\right)$ symmetric stretching at $1085 \mathrm{~cm}^{-1}$ to lower frequency. The bands assigned to proteins, lipids, nucleic acids, and hemoglobin (832, 925, 943, 1006, 1034, 1130, 1209, 1343 and $1607 \mathrm{~cm}^{-1}$ ) were used by Silveira et al. [44] for constructing an algorithm for skin cancer diagnosis. Based on Raman spectroscopy determinations of the relative concentrations of core biochemical tissue constituents (melanin, nucleic acid, elastin, ceramide, actin and phenylalanine), they demonstrated an efficient discrimination between healthy skin and pathological skin lesions (BCC and SCC). Statistically significant differences (ANOVA, $p<0.05$ ) between normal skin and BCC and normal skin and SCC were found for the relative concentration of melanin, DNA, actin and phenylalanine. Discriminant analysis with the Mahalanobis distance method (identification of outliers) of the melanin vs. phenylalanine yielded an overall accuracy of $72.3 \%$ when BCC and SCC were considered as a unique disease group by a sensitivity and specificity of $80.9 \%$ and $65.0 \%$ respectively. In a recent paper [45], the same research group, found that the spectra of the lesions exhibited higher Raman bands intensities compared with normal skin; BCC in the 1300-1700 $\mathrm{cm}^{-1}$ range, while SCC and AK in almost the whole spectral range. The difference spectra showed that BCC feature higher lipids concentration while the spectra of SCC suggest higher contribution from both lipids and proteins. Using partial least squares discriminant analysis (PLS-DA) they achieved $89.1 \%$ sensitivity and $94.3 \%$ specificity for the discrimination of BCC, SCC and AK from normal tissue and benign lesions. The PCA-DA analysis yielded $82.2 \%$ accuracy. Spectral vectors showed differences in the Amide III region attributed to pathologic expression of collagen and triolein. The amide III band was also considered as the most significant Raman spectral feature by Philipsen et al. [49] for the discrimination of BCC from MM. MM showed the highest intensity increase of the amide III band while BCC the lowest. Other studies however, showed that MM, unlike BCC, exhibit decrease in the intensity of the amide III band [37,60]. Moreover, the amide III ratio which is defined as the protein to lipid ratio for the bands near 1250 and $1300 \mathrm{~cm}^{-1}\left(\mathrm{I}_{\text {amide-III }} / \mathrm{I}_{1290-1330}\right)$ [70] was significantly different $(p=0.0075)$ between MM and BCC. No consistent spectral alterations were noticed in the amide I band region between tumor and normal skin. The authors presented two particularly interesting results from the analysis of skin lesions in vivo: (i) the clinical diagnostic efficacy of the Raman spectroscopy does not depend on the skin pigmentation and (ii) there is statistically significant $(p<0.05)$ higher intensity in the water band region in BCC and MM compared to normal skin. Based on their classification scheme (Mann-Whitney test), the diagnostic accuracy was MM 93.3\% vs. normal skin $96.4 \%$ and BCC $88.0 \%$ vs. normal skin $85.5 \%$. On the other hand, Schleusener et al. [41] did not notice any increase in the amide III band nor in the phenylalanine band $\left(\sim 1000 \mathrm{~cm}^{-1}\right)$, contrary to $[44,46]$. They also reported increased melanin content and decrease of the amide I band. Contrary to [46], their results supported a significant increase in the lipids content for $\mathrm{MM}$ in accordance to $[31,64]$. Using partial least squares discriminant analysis (PLS-DA), BCC and SCC were discriminated from normal skin with a balanced accuracy of $73 \%$ and $85 \%$, respectively. $\mathrm{MM}$ and pigmented nevi (PN) discrimination resulted in a balanced accuracy of 91\%. Lieber et al. [42] reported a $100 \%$ sensitivity and $91 \%$ specificity in the differentiation between healthy and malignant tissues (BCC and SCC) with a 95\% overall classification accuracy using a fiber-coupled portable Raman microscope. They suggested that the core differences in the Raman spectra between normal and 
malignant lesions mainly rely on divergent protein and lipid compositions of these tissues. Finally, Karagiannis et al. [51] developed a novel method where human MM cells were injected in mice. Tumor growth in this animal model was in vivo visualized by acoustic microscopy and analyzed by IR and Raman spectroscopy. Significant changes were observed in the $350-2000 \mathrm{~cm}^{-1}$ region of the Raman spectra.

\section{Conclusions}

In vivo Raman spectroscopy demonstrates significant potential as an emerging clinical diagnostic technique of high sensitivity and specificity for skin cancer screening. Raman spectroscopy requires no sample preparation and features minimal water interference; thus it can be successfully applied to non-invasive in vivo evaluation of skin lesions. Results show that real time Raman spectroscopy is able to provide high diagnostic accuracy with acceptable sensitivity and specificity for the effective detection of subtle biochemical alterations in skin tumors with adequate safety and without the need for any tissue pre-treatment. Nevertheless, experimental data show that there are no characteristic Raman peaks that can be uniquely assigned to a particular skin cancer type and significant variations can only be exposed by the application of statistical methods. The problem that multivariate analyses try to solve is two-fold: (i) to expose certain biochemical moieties that mainly contribute to the Raman signals of underline pathologies and (ii) to provide skin cancer screening tests with high sensitivity and specificity for the discrimination between normal and malignant tissues and between different skin lesions. This review showed that the complex environment of the skin tissue provides limited information on the former and complementary techniques may be beneficial. On the contrary, high discrimination accuracy is evident in most cases. The transition from laboratory bench to clinical bed-side setting still remains a challenging task that will take some time to mature. Several prerequisites have to be fulfilled, including optimizing diagnostic accuracy with minimal classification errors, adequate solutions for ethical prerequisites for the in vivo use, and fulfillment of all issues that guarantee complete compliance of the portable Raman spectrophotometers with the directives for medical devices. Finally, biopsy with histopathologic assessment, to date the reference procedure for the diagnosis of malignant skin lesions, cannot be replaced by any spectroscopic non-invasive approaches before large-scale comparative clinical trials have established their clinical confidence. Until then, real time, clinical acquisition of Raman spectra can play a supplementary but significant role towards optimizing skin tumor management.

\section{Acknowledgments}

This research project has been co-financed by the European Union (European Regional Development Fund-ERDF) and Greek national funds through the Operational Program “THESSALY-MAINLAND GREECE AND EPIRUS-2007-2013” of the National Strategic Reference Framework (NSRF 2007-2013). 


\section{Author Contributions}

Nikolaos Kourkoumelis conceived the study; Nikolaos Kourkoumelis, Ioannis Balatsoukas, Violetta Moulia and Aspasia Elka analyzed the data; Nikolaos Kourkoumelis, Georgios Gaitanis and Ioannis D. Bassukas wrote the manuscript.

\section{Conflicts of Interest}

The authors declare no conflict of interest.

\section{References}

1. Housman, T.S.; Feldman, S.R.; Williford, P.M.; Fleischer, A.B.; Goldman, N.D.; Acostamadiedo, J.M.; Chen, G.J. Skin cancer is among the most costly of all cancers to treat for the medicare population. $J$. Am. Acad. Dermatol. 2003, 48, 425-429.

2. Staples, M.P.; Elwood, M.; Burton, R.C.; Williams, J.L.; Marks, R.; Giles, G.G. Non-melanoma skin cancer in Australia: The 2002 national survey and trends since 1985. Med. J. Aust. 2006, 184, 6-10.

3. Rogers, H.W.; Weinstock, M.A.; Harris, A.R.; Hinckley, M.R.; Feldman, S.R.; Fleischer, A.B.; Coldiron, B.M. Incidence estimate of nonmelanoma skin cancer in the united states, 2006. Arch. Dermatol. 2010, 146, 283-287.

4. Brewster, D.H.; Bhatti, L.A.; Inglis, J.H.C.; Nairn, E.R.; Doherty, V.R. Recent trends in incidence of nonmelanoma skin cancers in the east of Scotland, 1992-2003. Brit. J. Dermatol. 2007, 156, 1295-1300.

5. Hoey, S.E.H.; Devereux, C.E.J.; Murray, L.; Catney, D.; Gavin, A.; Kumar, S.; Donnelly, D.; Dolan, O.M. Skin cancer trends in northern Ireland and consequences for provision of dermatology services. Br. J. Dermatol. 2007, 156, 1301-1307.

6. Donaldson, M.R.; Coldiron, B.M. No end in sight: The skin cancer epidemic continues. Semin. Cutan. Med. Surg. 2011, 30, 3-5.

7. Sng, J.; Koh, D.; Siong, W.C.; Choo, T.B. Skin cancer trends among Asians living in Singapore from 1968 to 2006. J. Am. Acad. Dermatol. 2009, 61, 426-432.

8. Gandini, S.; Sera, F.; Cattaruzza, M.S.; Pasquini, P.; Abeni, D.; Boyle, P.; Melchi, C.F. Meta-analysis of risk factors for cutaneous melanoma: I. Common and atypical naevi. Eur. J. Cancer 2005, 41, $28-44$.

9. Forchetti, G.; Suppa, M.; del Marmol, V. Overview on non-melanoma skin cancers in solid organ transplant recipients. G. Ital. Dermatol. V 2014, 149, 383-387.

10. Simoes, M.C.F.; Sousa, J.J.S.; Pais, A.A.C.C. Skin cancer and new treatment perspectives: A review. Cancer Lett. 2015, 357, 8-42.

11. Jensen, A.O.; Bautz, A.; Olesen, A.B.; Karagas, M.R.; Sorensen, H.T.; Friis, S. Mortality in Danish patients with nonmelanoma skin cancer, 1978-2001. Br. J. Dermatol. 2008, 159, 419-425.

12. Lewis, K.G.; Weinstock, M.A. Nonmelanoma skin cancer mortality (1988-2000): The Rhode Island follow-back study. Arch. Dermatol. 2004, 140, 837-842.

13. Hannuksela-Svahn, A.; Pukkala, E.; Karvonen, J. Basal cell skin carcinoma and other nonmelanoma skin cancers in Finland from 1956 through 1995. Arch. Dermatol. 1999, 135, 781-786. 
14. Bassukas, I.D.; Lippold, A.; Hundeiker M. Parametric estimation of the "risk of melanoma-related death" with the recursion formula of the gompertz survival function. In Skin Cancer and UV Radiation; Altmeyer, P., Hoffmann, K., Stücker, M., Eds.; Springer: Berlin, Germany; Heidelberg, Germany, 1997; pp. 527-533.

15. Patil, C.A.; Kirshnamoorthi, H.; Ellis, D.L.; van Leeuwen, T.G.; Mahadevan-Jansen, A. A clinical instrument for combined Raman spectroscopy-optical coherence tomography of skin cancers. Laser Surg. Med. 2011, 43, 143-151.

16. Mordechai, S.; Sahu, R.K.; Hammody, Z.; Mark, S.; Kantarovich, K.; Guterman, H.; Podshyvalov, A.; Goldstein, J.; Argov, S. Possible common biomarkers from FTIR microspectroscopy of cervical cancer and melanoma. J. Microsc. 2004, 215, 86-91.

17. Baranska, M.; Byrne, H.J. Optical diagnostics-spectropathology for the next generation. Analyst 2015, 140, 2064-2065.

18. Kourkoumelis, N. Spectroscopy for biosciences. Contemp. Phys. 2015, doi:10.1080/00107514. 2015.1006254.

19. Bigio, I.J.; Mourant, J.R. Ultraviolet and visible spectroscopies for tissue diagnostics: Fluorescence spectroscopy and elastic-scattering spectroscopy. Phys. Med. Biol. 1997, 42, 803-814.

20. Seddon, A.B. Mid-infrared (IR) - A hot topic: The potential for using mid-IR light for non-invasive early detection of skin cancer in vivo. Phys. Status Solidi 2013, 250, 1020-1027.

21. Kendall, C.; Isabelle, M.; Bazant-Hegemark, F.; Hutchings, J.; Orr, L.; Babrah, J.; Baker, R.; Stone, N. Vibrational spectroscopy: A clinical tool for cancer diagnostics. Analyst 2009, 134, 1029-1045.

22. Chan, J.W.; Lieu, D.K. Label-free biochemical characterization of stem cells using vibrational spectroscopy. J. Biophotonics 2009, 2, 656-668.

23. Kong, K.; Kendall, C.; Stone, N.; Notingher, I. Raman spectroscopy for medical diagnostics_-From in-vitro biofluid assays to in-vivo cancer detection. Adv. Drug Deliv. Rev. 2015, in press.

24. Kourkoumelis, N.; Polymeros, A.; Tzaphlidou, M. Background estimation of biomedical Raman spectra using a geometric approach. J. Spectrosc. 2012, 27, 441-447.

25. Devpura, S.; Barton, K.N.; Brown, S.L.; Palyvoda, O.; Kalkanis, S.; Naik, V.M.; Siddiqui, F.; Naik, R.; Chetty, I.J. Vision 20/20: The role of Raman spectroscopy in early stage cancer detection and feasibility for application in radiation therapy response assessment. Med. Phys. 2014, 41, doi:10.1118/1.4870981.

26. Wang, W.; Zhao, J.; Short, M.; Zeng, H. Real-time in vivo cancer diagnosis using Raman spectroscopy. J. Biophotonics 2014, doi:10.1002/jbio.201400026.

27. Vandenabeele, P.; Jehlicka, J.; Vitek, P.; Edwards, H.G.M. On the definition of Raman spectroscopic detection limits for the analysis of biomarkers in solid matrices. Planet. Space Sci. 2012, 62, 48-54.

28. Tfaili, S.; Josse, G.; Gobinet, C.; Angiboust, J.F.; Manfait, M.; Piot, O. Shedding light on the laser wavelength effect in Raman analysis of skin epidermises. Analyst 2012, 137, 4241-4246.

29. Naito, S.; Min, Y.K.; Sugata, K.; Osanai, O.; Kitahara, T.; Hiruma, H.; Hamaguchi, H. In vivo measurement of human dermis by 1064 nm-excited fiber Raman spectroscopy. Skin Res. Technol. 2008, 14, 18-25.

30. Patil, C.A.; Pence, I.J.; Lieber, C.A.; Mahadevan-Jansen, A. $1064 \mathrm{~nm}$ dispersive Raman spectroscopy of tissues with strong near-infrared autofluorescence. Opt. Lett. 2014, 39, 303-306. 
31. Fendel, S.; Schrader, B. Investigation of skin and skin lesions by NIR-FT-Raman spectroscopy. Fresenius J. Anal. Chem. 1998, 360, 609-613.

32. Franzen, L.; Selzer, D.; Fluhr, J.W.; Schaefer, U.F.; Windbergs, M. Towards drug quantification in human skin with confocal Raman microscopy. Eur. J. Pharm. Biopharm. 2013, 84, 437-444.

33. Tfaili, S.; Gobinet, C.; Josse, G.; Angiboust, J.F.; Baillet, A.; Manfait, M.; Piot, O. Vibrational spectroscopies for the analysis of cutaneous permeation: Experimental limiting factors identified in the case of caffeine penetration. Anal. Bioanal. Chem. 2013, 405, 1325-1332.

34. Tfayli, A.; Piot, O.; Pitre, F.; Manfait, M. Follow-up of drug permeation through excised human skin with confocal Raman microspectroscopy. Eur. Biophys. J. Biophys. 2007, 36, 1049-1058.

35. Franzen, L.; Anderski, J.; Planz, V.; Kostka, K.H.; Windbergs, M. Combining confocal Raman microscopy and freeze-drying for quantification of substance penetration into human skin. Exp. Dermatol. 2014, 23, 942-944.

36. Lui, H.; Zhao, J.H.; McLean, D.; Zeng, H.S. Real-time Raman spectroscopy for in vivo skin cancer diagnosis. Cancer Res. 2012, 72, 2491-2500.

37. Zakharov, V.P.; Bratchenko, I.A.; Kozlov, S.V.; Moryatov, A.A.; Myakinin, O.O.; Artemyev, D.N. Two-step Raman spectroscopy method for tumor diagnosis. In Proceedings of the SPIE 9129, Biophotonics: Photonic Solutions for Better Health Care IV, Brussels, Belgium, 14-17 April 2014; doi:10.1117/12.2052121.

38. Zakharov, V.P.; Bratchenko, I.A.; Kozlov, S.V.; Moryatov, A.A.; Kornilin, D.V.; Myakinin, O.O.; Artemyev, D.N. Advances in tumor diagnosis using OCT and Raman spectroscopy. In Proceedings of the SPIE 9129, Biophotonics: Photonic Solutions for Better Health Care IV, Brussels, Belgium, 14-17 April 2014; doi:10.1117/12.2052145.

39. Zakharov, V.P.; Bratchenko, I.A.; Artemyev, D.N.; Myakinin, O.O.; Kornilin, D.V.; Kozlov, S.V.; Moryatov, A.A. Comparative analysis of combined spectral and optical tomography methods for detection of skin and lung cancers. J. Biomed. Opt. 2015, 20, doi:10.1117/1.JBO.20.2.025003.

40. Schleusener, J.; Reble, C.; Helfmann, J.; Gersonde, I.; Cappius, H.J.; Glanert, M.; Fluhr, J.W.; Meinke, M.C. Design and technical evaluation of fibre-coupled Raman probes for the image-guided discrimination of cancerous skin. Meas. Sci. Technol. 2014, 25, doi:10.1088/0957-0233/25/3/035701.

41. Schleusener, J.; Gluszczynska, P.; Reble, C.; Gersonde, I.; Helfmann, J.; Fluhr, J.W.; Lademann, J.; Röwert-Huber, J.; Meinke, M.C. In vivo study for the discrimination of cancerous and normal skin using fiber probe based Raman spectroscopy. Exp. Dermatol. 2015, in press.

42. Lieber, C.A.; Majumder, S.K.; Ellis, D.L.; Billheimer, D.D.; Mahadevan-Jansen, A. In vivo nonmelanoma skin cancer diagnosis using Raman microspectroscopy. Laser Surg. Med. 2008, 40, 461-467.

43. Tfayli, A.; Piot, O.; Derancourt, S.; Cadiot, G.; Diebold, M.D.; Bernard, P.; Manfait, M. In vivo analysis of tissue by Raman microprobe: Examination of human skin lesions and esophagus Barrett's mucosa on an animal model. In Proceedings of the SPIE 6093, Biomedical Vibrational Spectroscopy III: Advances in Research and Industry, San Jose, CA, USA, 27 February 2006; doi:10.1117/12.645874.

44. Silveira, F.L.; Pacheco, M.T.T.; Bodanese, B.; Zângaro, R.A.; Silveira, L. Discriminating model for skin cancer diagnosis in vivo through Raman spectroscopy. In Proceedings of the SPIE 8565, Photonic Therapeutics and Diagnostics IX, San Francisco, CA, USA, 2 February 2013; doi:10.1117/12.2007293. 
45. Silveira, F.L.; Pacheco, M.T.; Bodanese, B.; Pasqualucci, C.A.; Zangaro, R.A.; Silveira, L., Jr. Discrimination of non-melanoma skin lesions from non-tumor human skin tissues in vivo using Raman spectroscopy and multivariate statistics. Lasers Surg. Med. 2015, 47, 6-16.

46. Lim, L.; Nichols, B.; Migden, M.R.; Rajaram, N.; Reichenberg, J.S.; Markey, M.K.; Ross, M.I.; Tunnell, J.W. Clinical study of noninvasive in vivo melanoma and nonmelanoma skin cancers using multimodal spectral diagnosis. J. Biomed. Opt. 2014, 19, doi:10.1117/1.JBO.19.11.117003.

47. Motz, J.T.; Gandhi, S.J.; Scepanovic, O.R.; Haka, A.S.; Kramer, J.R.; Dasari, R.R.; Feld, M.S. Real-time raman system for in vivo disease diagnosis. J. Biomed. Opt. 2005, 10, doi:10.1117/1.1920247.

48. Sharma, M.; Marple, E.; Reichenberg, J.; Tunnell, J.W. Design and characterization of a novel multimodal fiber-optic probe and spectroscopy system for skin cancer applications. Rev. Sci. Instrum. 2014, 85, doi:10.1063/1.4890199.

49. Philipsen, P.A.; Knudsen, L.; Gniadecka, M.; Ravnbak, M.H.; Wulf, H.C. Diagnosis of malignant melanoma and basal cell carcinoma by in vivo NIR-FT Raman spectroscopy is independent of skin pigmentation. Photoch. Photobiol. Sci. 2013, 12, 770-776.

50. Hata, T.R.; Scholz, T.A.; Ermakov, I.V.; McClane, R.W.; Khachik, F.; Gellermann, W.; Pershing, L.K. Non-invasive Raman spectroscopic detection of carotenoids in human skin. J. Investig. Dermatol. 2000, 115, 441-448.

51. Karagiannis, G.T.; Grivas, I.; Tsingotjidou, A.; Apostolidis, G.K.; Grigoriadou, I.; Dori, I.; Poulatsidou, K.-N.; Doumas, A.; Wesarg, S.; Georgoulias, P. Early detection of melanoma with the combined use of acoustic microscopy, infrared reflectance and Raman spectroscopy. In Proceedings of the SPIE 9323, Photons Plus Ultrasound: Imaging and Sensing, San Francisco, CA, USA, 13-18 February 2015; doi:10.1117/12.2079690.

52. Lieber, C.A.; Majumder, S.K.; Billheimer, D.; Ellis, D.L.; Mahadevan-Jansen, A. Raman microspectroscopy for skin cancer detection in vitro. J. Biomed. Opt. 2008, 13, doi:10.1117/ 1.2899155 .

53. Zheng, J.K.; Pang, S.; Labuza, T.P.; He, L.L. Evaluation of surface-enhanced raman scattering detection using a handheld and a bench-top Raman spectrometer: A comparative study. Talanta 2014, 129, 79-85.

54. Mogensen, M.; Jemec, G.B.E. Diagnosis of nonmelanoma skin cancer/keratinocyte carcinoma: A review of diagnostic accuracy of nonmelanoma skin cancer diagnostic tests and technologies. Dermatol. Surg. 2007, 33, 1158-1174.

55. Hanlon, E.B.; Manoharan, R.; Koo, T.W.; Shafer, K.E.; Motz, J.T.; Fitzmaurice, M.; Kramer, J.R.; Itzkan, I.; Dasari, R.R.; Feld, M.S. Prospects for in vivo Raman spectroscopy. Phys. Med. Biol. 2000, 45, R1-R59.

56. Nijssen, A.; Schut, T.C.B.; Heule, F.; Caspers, P.J.; Hayes, D.P.; Neumann, M.H.A.; Puppels, G.J. Discriminating basal cell carcinoma from its surrounding tissue by Raman spectroscopy. J. Investig. Dermatol. 2002, 119, 64-69.

57. Kong, K.; Rowlands, C.J.; Varma, S.; Perkins, W.; Leach, I.H.; Koloydenko, A.A.; Pitiot, A.; Williams, H.C.; Notingher, I. Increasing the speed of tumour diagnosis during surgery with selective scanning Raman microscopy. J. Mol. Struct. 2014, 1073, 58-65.

58. Ly, E.; Piot, O.; Durlach, A.; Bernard, P.; Manfait, M. Polarized Raman microspectroscopy can reveal structural changes of peritumoral dermis in basal cell carcinoma. Appl. Spectrosc. 2008, 62, 1088-1094. 
59. Choi, J.; Choo, J.; Chung, H.; Gweon, D.G.; Park, J.; Kim, H.J.; Park, S.; Oh, C.H. Direct observation of spectral differences between normal and basal cell carcinoma (BCC) tissues using confocal Raman microscopy. Biopolymers 2005, 77, 264-272.

60. Gniadecka, M.; Philipsen, P.A.; Sigurdsson, S.; Wessel, S.; Nielsen, O.F.; Christensen, D.H.; Hercogova, J.; Rossen, K.; Thomsen, H.K.; Gniadecki, R.; et al. Melanoma diagnosis by Raman spectroscopy and neural networks: Structure alterations in proteins and lipids in intact cancer tissue. J. Investig. Dermatol. 2004, 122, 443-449.

61. Larraona-Puy, M.; Ghita, A.; Zoladek, A.; Perkins, W.; Varma, S.; Leach, I.H.; Koloydenko, A.A.; Williams, H.; Notingher, I. Development of Raman microspectroscopy for automated detection and imaging of basal cell carcinoma. J. Biomed. Opt. 2009, 14, doi:10.1117/1.3251053.

62. Gniadecka, M.; Wulf, H.C.; Mortensen, N.N.; Nielsen, O.F.; Christensen, D.H. Diagnosis of basal cell carcinoma by Raman spectroscopy. J. Raman Spectrosc. 1997, 28, 125-129.

63. Dukor, R.K. Vibrational spectroscopy in the detection of cancer. In Handbook of Vibrational Spectroscopy; Chalmers, J., Griffiths, P.R., Eds; John Wiley and Sons Ltd.: Hoboken, NJ, USA, 2002; Volume 5, pp. 3335-3361.

64. Cartaxo, S.B.; Santos, I.D.D.O.; Bitar, R.; Oliveira, A.F.; Ferreira, L.M.; Martinho, H.S.; Martin, A.A. FT-Raman spectroscopy for the differentiation between cutaneous melanoma and pigmented nevus. Acta Cir. Bras. 2010, 25, 351-356.

65. Darvin, M.E.; Sterry, W.; Lademann, J.; Vergou, T. The role of carotenoids in human skin. Molecules 2011, 16, 10491-10506.

66. Lademann, J.; Kocher, W.; Yu, R.; Meinke, M.C.; Na Lee, B.; Jung, S.; Sterry, W.; Darvin, M.E. Cutaneous carotenoids: The mirror of lifestyle? Skin Pharmacol. Physiol. 2014, 27, doi:10.1159/ 000357222.

67. Chen, L.; Wang, Y.; Liu, N.; Lin, D.; Weng, C.; Zhang, J.; Zhu, L.; Chen, W.; Chen, R.; Feng, S. Near-infrared confocal micro-Raman spectroscopy combined with PCA-LDA multivariate analysis for detection of esophageal cancer. Laser Phys. 2013, 23, doi:10.1088/1054-660X/23/6/065601.

68. Zhao, J.; Huang, Z.; Zeng, H.; McLean, D.I.; Lui, H. Quantitative analysis of skin chemicals using rapid near-infrared Raman spectroscopy. In Proceedings of the SPIE 6842, Photonic Therapeutics and Diagnostics IV, San Jose, CA, USA, 19 January 2008; doi:10.1117/12.769373.

69. Salomatina, E.; Jiang, B.; Novak, J.; Yaroslavsky, A.N. Optical properties of normal and cancerous human skin in the visible and near-infrared spectral range. J. Biomed. Opt. 2006, 11, doi:10.1117/1.2398928.

70. Bodanese, B.; Silveira, L.; Albertini, R.; Zangaro, R.A.; Pacheco, M.T.T. Differentiating normal and basal cell carcinoma human skin tissues in vitro using dispersive Raman spectroscopy: A comparison between principal components analysis and simplified biochemical models. Photomed. Laser Surg. 2010, 28, S119-S127.

(C) 2015 by the authors; licensee MDPI, Basel, Switzerland. This article is an open access article distributed under the terms and conditions of the Creative Commons Attribution license (http://creativecommons.org/licenses/by/4.0/). 